\title{
WNT5A enhances resistance of melanoma cells to targeted BRAF inhibitors
}

\author{
Jamie N. Anastas, ${ }^{1,2}$ Rima M. Kulikauskas, ${ }^{3}$ Tigist Tamir, ${ }^{4}$ Helen Rizos, ${ }^{5}$ Georgina V. Long, ${ }^{5}$ \\ Erika M. von Euw, ${ }^{6}$ Pei-Tzu Yang, ${ }^{2}$ Hsiao-Wang Chen, ${ }^{6}$ Lauren Haydu, ${ }^{5}$ Rachel A. Toroni, ${ }^{3}$ \\ Olivia M. Lucero, ${ }^{2,3}$ Andy J. Chien, ${ }^{2,3}$ and Randall T. Moon ${ }^{2}$
}

\begin{abstract}
${ }^{1}$ Molecular Cell Biology Graduate Program, University of Washington, Seattle, Washington, USA. 2Department of Pharmacology, Howard Hughes Medical Institute, Institute for Stem Cell and Regenerative Medicine, ${ }^{3}$ Division of Dermatology, Department of Medicine, University of Washington, Seattle, Washington, USA. ${ }^{4}$ HHMI EXROP Summer Research Program, University of Washington School of Medicine, Seattle, Washington, USA. ${ }^{5}$ Westmead Institute for Cancer Research, Westmead Hospital, The University of Sydney and the Melanoma Institute Australia, Sydney, New South Wales, Australia. ${ }^{6}$ UCLA JCCC-Translational Oncology Research Labs, Los Angeles, California, USA.
\end{abstract}

\begin{abstract}
About half of all melanomas harbor a mutation that results in a constitutively active BRAF kinase mutant $\left(\mathrm{BRAF}^{\mathrm{V} 600 \mathrm{E} / \mathrm{K}}\right.$ ) that can be selectively inhibited by targeted BRAF inhibitors (BRAFis). While patients treated with BRAFis initially exhibit measurable clinical improvement, the majority of patients eventually develop drug resistance and relapse. Here, we observed marked elevation of WNT5A in a subset of tumors from patients exhibiting disease progression on BRAFi therapy. WNT5A transcript and protein were also elevated in BRAFiresistant melanoma cell lines generated by long-term in vitro treatment with BRAFi. RNAi-mediated reduction of endogenous WNT5A in melanoma decreased cell growth, increased apoptosis in response to BRAFi challenge, and decreased the activity of prosurvival AKT signaling. Conversely, overexpression of WNT5A promoted melanoma growth, tumorigenesis, and activation of AKT signaling. Similarly to WNT5A knockdown, knockdown of the WNT receptors FZD7 and RYK inhibited growth, sensitized melanoma cells to BRAFi, and reduced AKT activation. Together, these findings suggest that chronic BRAF inhibition elevates WNT5A expression, which promotes AKT signaling through FZD7 and RYK, leading to increased growth and therapeutic resistance. Furthermore, increased WNT5A expression in BRAFi-resistant melanomas correlates with a specific transcriptional signature, which identifies potential therapeutic targets to reduce clinical BRAFi resistance.
\end{abstract}

\section{Introduction}

Over two-thirds of melanomas exhibit activating mutations in the MAPK pathway-activating enzymes NRAS and BRAF, which include $B R A F^{V 600 E}$ and to a lesser extent $B_{R A F^{V 600 K}}(1,2)$. Small molecules such as vemurafenib and dabrafenib have been developed to specifically target mutant BRAFV600E, although they also exhibit activity against $\operatorname{BRAF}^{\mathrm{V} 600 \mathrm{~K}}(3,4)$. Use of these BRAF inhibitors (BRAFis) in metastatic melanoma patients with activating $B R A F$ mutations has resulted in unprecedented decreases in tumor burden and improvement in overall survival (5-7). Unfortunately, the majority of patients develop resistance to BRAFis and subsequently relapse (5-8). Studies to date on BRAFi-resistant melanoma cells have focused largely on reactivation of the MAPK signaling pathway downstream of BRAF through somatic mutations in MAPK pathway members including $M E K$ and $\operatorname{NRAS}(9,10)$, and by splice variants in $B R A F(11)$. While patients on combination therapies involving a BRAFi plus a MEK inhibitor (MEKi) exhibit significantly higher response rates than patients on a BRAFi alone, the observation of resistance in patients on combined BRAFi/MEKi therapy suggests that factors independent of MAPK reactivation are also involved in the development of therapeutic resistance (12, 13). For instance, BRAFi-resistant melanoma cells exhibit aberrations in the expression or activity of receptor tyrosine kinases such as PDGFR $\beta$, VEGFR, EGFR, and $\operatorname{IGFR}(9,14,15)$, as well as changes in the expression of pro- and antiapoptotic proteins (16-18).

The WNT gene family encodes secreted proteins that act as ligands to stimulate $\beta$-catenin-dependent and $\beta$-catenin-independent sig-

Conflict of interest: The authors have declared that no conflict of interest exists. Citation for this article: J Clin Invest. 2014;124(7):2877-2890. doi:10.1172/JCI70156. naling pathways. While most cancer research has focused on the WNT/ $\beta$-catenin signaling pathway, numerous studies now identify aberrations in $\beta$-catenin-independent WNT pathways in melanoma, most notably those activated by WNT5A $(19,20)$.

Recent studies have reported that altered WNT/ $\beta$-catenin signaling can change the sensitivity of tumor cells to therapeutic drugs (21-24), yet potential roles for $\beta$-catenin-independent WNT signaling in drug resistance are not well understood. In the present study, we found that WNT5A protein and transcript levels were dramatically increased in BRAFi-resistant cell lines and in patient tumors. Functional studies revealed that endogenous WNT5A was required for the growth and survival of naive melanoma cells that had not been exposed to BRAFi. WNT5A was also required for the proliferation and survival of melanoma cells that have acquired resistance to BRAFis due to chronic drug treatment. In pursuing the underlying mechanisms that might account for these effects of WNT5A on melanoma cells, we found that WNT5A activated PI3K/AKT signaling, another critical pathway in melanoma proliferation and resistance to apoptosis $(25,26)$. Further mechanistic studies suggested that WNT5A promotes melanoma growth and survival via its receptors RYK and FZD7. Collectively, these studies revealed an unsuspected role for WNT5A-dependent signaling in promoting the resistance of melanoma cells to BRAFis.

\section{Results}

Chronic inhibition of BRAF ${ }^{V 600 E}$ with PLX4720 elevates WNT5A expression. Previous expression microarray profiling revealed that melanoma cell lines inherently insensitive to low doses of a BRAFi (PLX4032) express elevated levels of WNT5A (27). In the present study, we first asked whether WNT5A expression is similarly increased in melanoma cells exhibiting de novo resistance to chronic inhibition of 

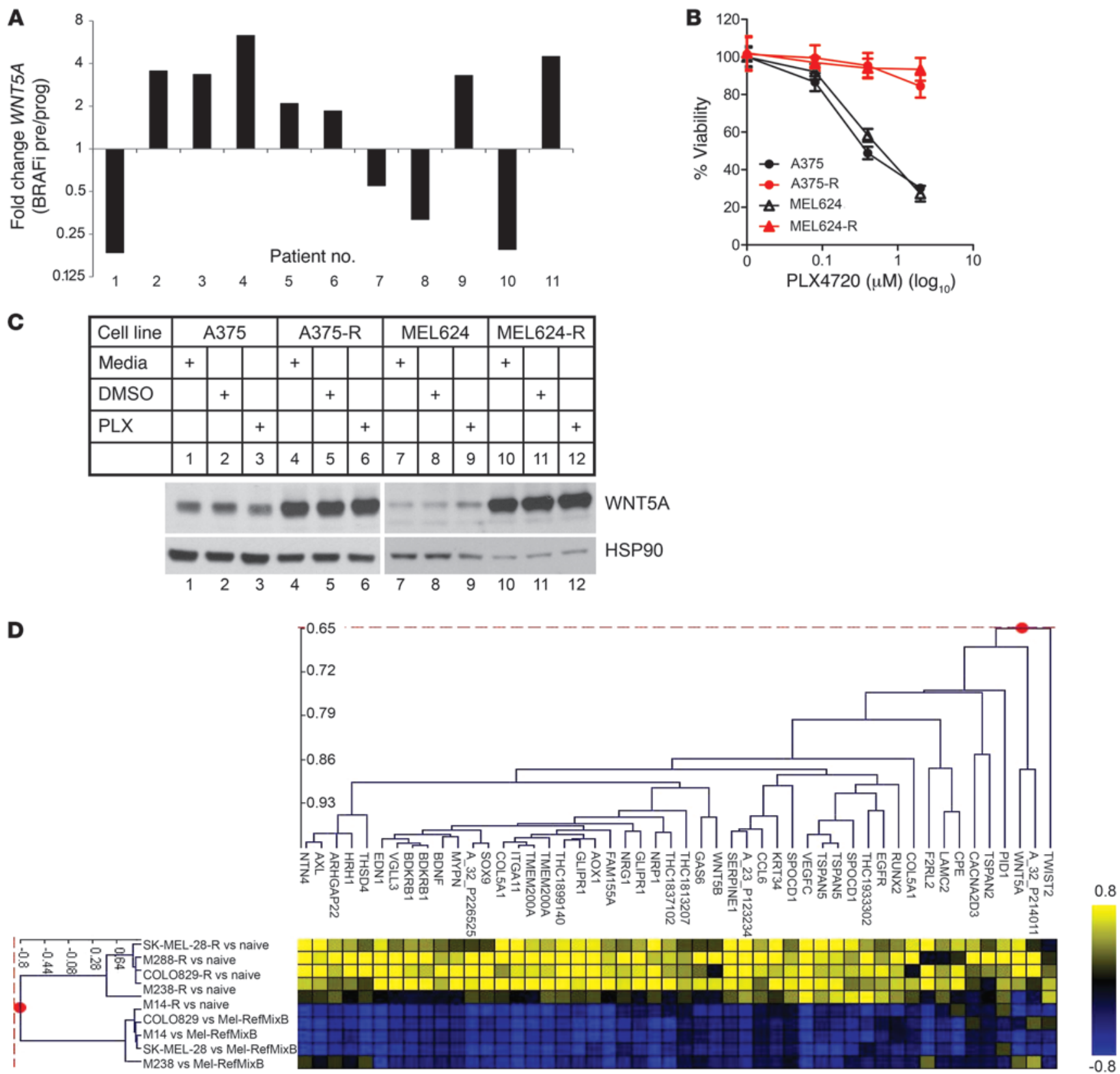

Figure 1

WNT5A expression is increased in response to chronic inhibition of BRAFV600E with PLX4720. (A) Fold change in WNT5A mRNA expression normalized to GAPDH in BRAFi-resistant patient tumors (as marked by disease progression [prog]) compared with paired tumor biopsies collected before the start of treatment (pre). (B) Normalized viability of A375 and MEL624 parental and PLX-resistant cells (-R) after treatment with either medium, DMSO, or increasing doses of PLX4720 for 48 hours. Resistant cell lines (A375-R and MEL624-R) were derived by treatment of melanoma cells with $2 \mu \mathrm{M}$ PLX4720 for more than 10 weeks. (C) Western blots of lysates from A375, A375-R, MEL624, and MEL624-R cells after either culturing in standard growth medium or treatment with vehicle control (DMSO) or $2 \mu \mathrm{M}$ PLX4720 for 2 days. (D) Hierarchical clustering of gene expression in PLX-resistant $(-R)$ and PLX-naive cells displaying the subset of genes that significantly correlate with WNT5A expression. The top 5 rows represent relative expression changes in naive cell lines compared with BRAFi-resistant (-R) cell lines generated by chronic treatment with PLX4032. The lower 5 rows display the relative expression of these genes in naive melanoma cells in comparison with a reference data set determined as an average of the expression profiles of 47 different melanoma cell lines (Mel-RefMixB).

BRAF $^{\mathrm{V} 600 \mathrm{E}}$. Specifically, we used real-time quantitative RT-PCR (qRT-PCR) to monitor the relative levels of WNT5A transcripts in melanoma tumors from patients who developed resistance to $\mathrm{BRAF}^{\mathrm{V} 600 \mathrm{E}}$-targeted therapies (as measured by disease progression) and compared expression with samples taken before the start of treatment. We found that WNT5A expression was increased in a subset of BRAFi-resistant patient tumors (7 of 11) compared with pretreated samples (Figure 1A). In this small patient cohort, we did not observe a significant association between WNT5A expression and either melanoma-specific survival $(P=0.059)$ or progression- 

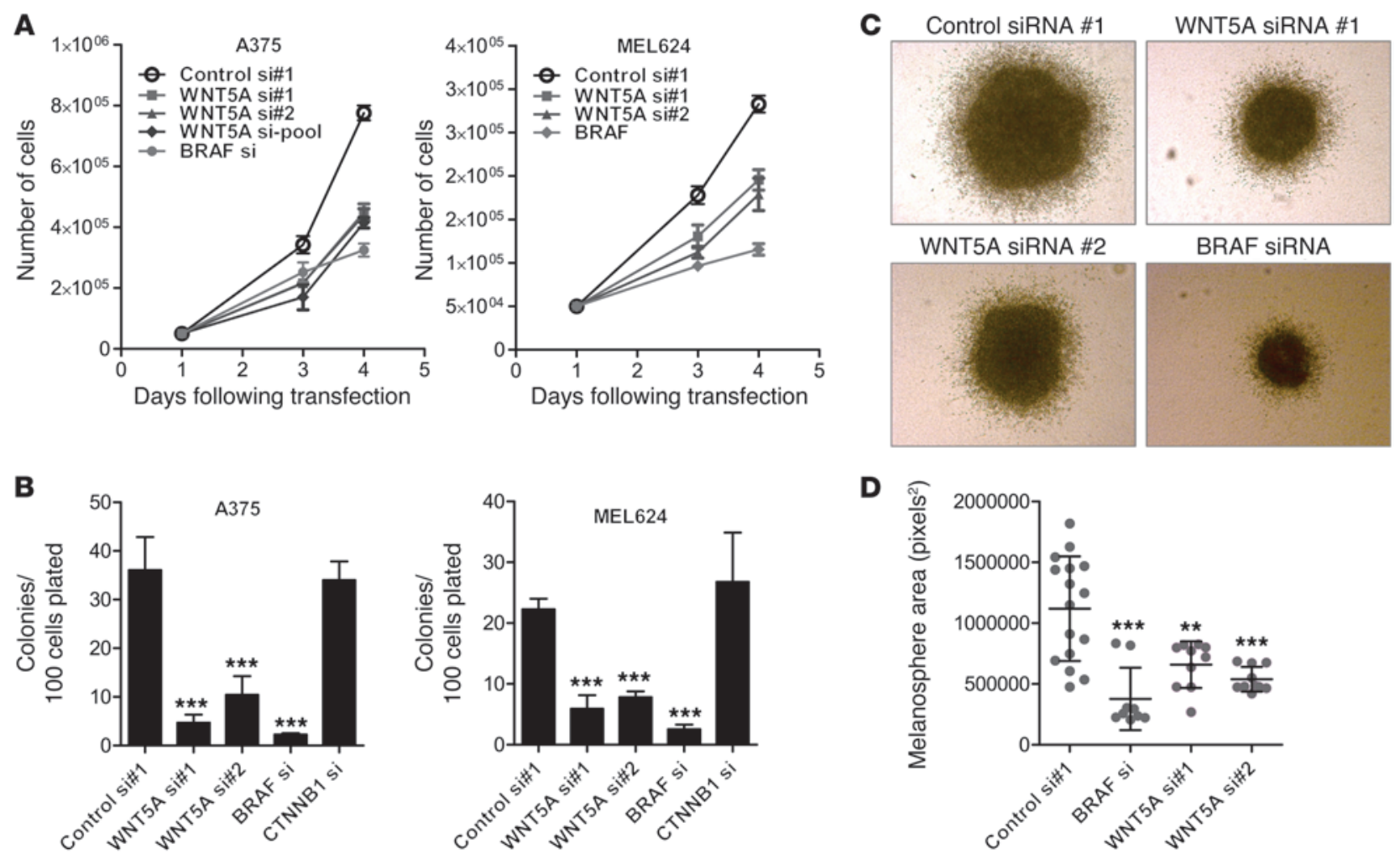

Figure 2

WNT5A loss of function reduces melanoma cell number. (A) Growth curves of A375 (left) and MEL624 (right) melanoma cells transfected with control siRNA \#1, WNT5A siRNAs \#1 and \#2, and BRAF siRNA as a positive control. Error bars indicate SD calculated from 3 independent experiments with triplicate wells counted for each time point. (B) Average number of colonies per 100 A375 (left) or MEL624 (right) melanoma cells plated in soft agar, scored 1 week after siRNA transfection and embedding in soft agar. Error bars indicate SD calculated from 3 independent experiments with duplicate wells for each siRNA. (C) Representative photographs of A375 cells grown in 3D Collagen I culture after siRNA transfection. (D) Quantification of A375 spheroid area at day 4 following siRNA transfection. Each point represents the area of a single melanosphere determined by image analysis. Center bars indicates the mean melanosphere area, and error bars show the SD. See Methods for further details on measurement procedure. Statistically significant differences in colony formation in soft agar and in melanosphere size were calculated using 1-way ANOVA. ${ }^{\star *} P<0.01,{ }^{\star \star *} P<0.001$.)

free survival $(P=0.068)$. Another independent study has similarly observed increased WNT5A expression in patient melanomas exhibiting resistance to BRAFi, suggesting that upregulation of WNT5A is a common molecular aberration in drug-resistant melanoma (28).

To investigate the potential consequences of increased WNT5A in BRAFi-resistant melanomas, we next established BRAFi-resistant melanoma cell lines in culture. A375 and MEL624 human melanoma cells were treated with $2 \mu \mathrm{M}$ of the BRAFi PLX 4720 for more than 10 weeks to generate "resistant" cell lines (subsequently referred to as A375-R and MEL624-R). We validated the resistance of these cells to BRAFi by treating equal numbers of parental and resistant cells with increasing doses of BRAFi for 3 days and then determining cell viability. BRAFi treatment decreased the viability of parental cells in a dose-dependent manner as previously reported, yet did not affect the growth of either A375-R or MEL624-R cells (Figure 1B), confirming the insensitivity of these cells to BRAFi treatment.

We then analyzed the expression of WNT5A protein in both parental and resistant cell lines. Immunoblots revealed a dramatic increase in levels of WNT5A in the resistant cells (Figure 1C). Returning the resistant cells to either normal medium or medium containing vehicle (DMSO) did not diminish the high levels of
WNT5A observed in BRAFi-resistant samples (Figure 1C), suggesting that the upregulation of WNT5A in resistant cells does not require the continual presence of BRAFi. WNT5A protein was similarly increased following knockdown of endogenous BRAF with siRNA (Supplemental Figure 1A; supplemental material available online with this article; doi:10.1172/JCI70156DS1), indicating that increased WNT5A expression is a consequence of loss of BRAF activity rather than an off-target effect of PLX4720 treatment.

We next asked whether WNT5A levels are increased in additional melanoma cell lines as a consequence of long-term BRAFV600E inhibition. We used microarray profiling to analyze changes in gene expression in COLO829, SK-MEL-28, M288, M238, and M14 melanoma cell lines chronically treated with BRAFi (PLX4032). Although baseline expression of WNT5A varied between different melanoma cell lines (Supplemental Figure 1B), chronic treatment with BRAFi correlated with elevated WNT5A in 4 of 5 additional cell lines tested (Figure 1D). We further analyzed the expression profiles of these BRAFi-resistant cell lines using hierarchical clustering in order to identify additional transcripts with expression profiles similar to that of WNT5A. The top 50 genes significantly correlating with WNT5A expression included transcription factors 
A

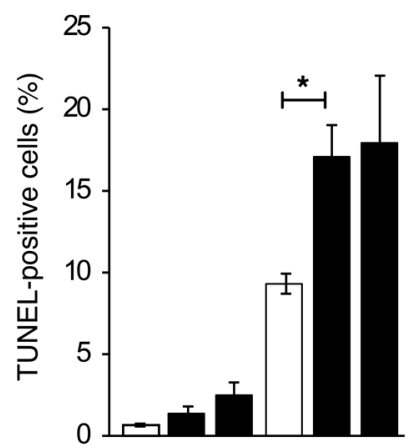

\begin{tabular}{|r|r|r|r|r|r|r|}
\cline { 2 - 6 } \multicolumn{1}{c|}{ Treatment: } & \multicolumn{3}{|c|}{ DMSO } & \multicolumn{3}{c|}{ PLX } \\
\hline Control si\#1 & + & & & + & & \\
\hline WNT5A si\#1 & & + & & & + & \\
\hline WNT5A si\#2 & & & + & & & + \\
\hline
\end{tabular}

B
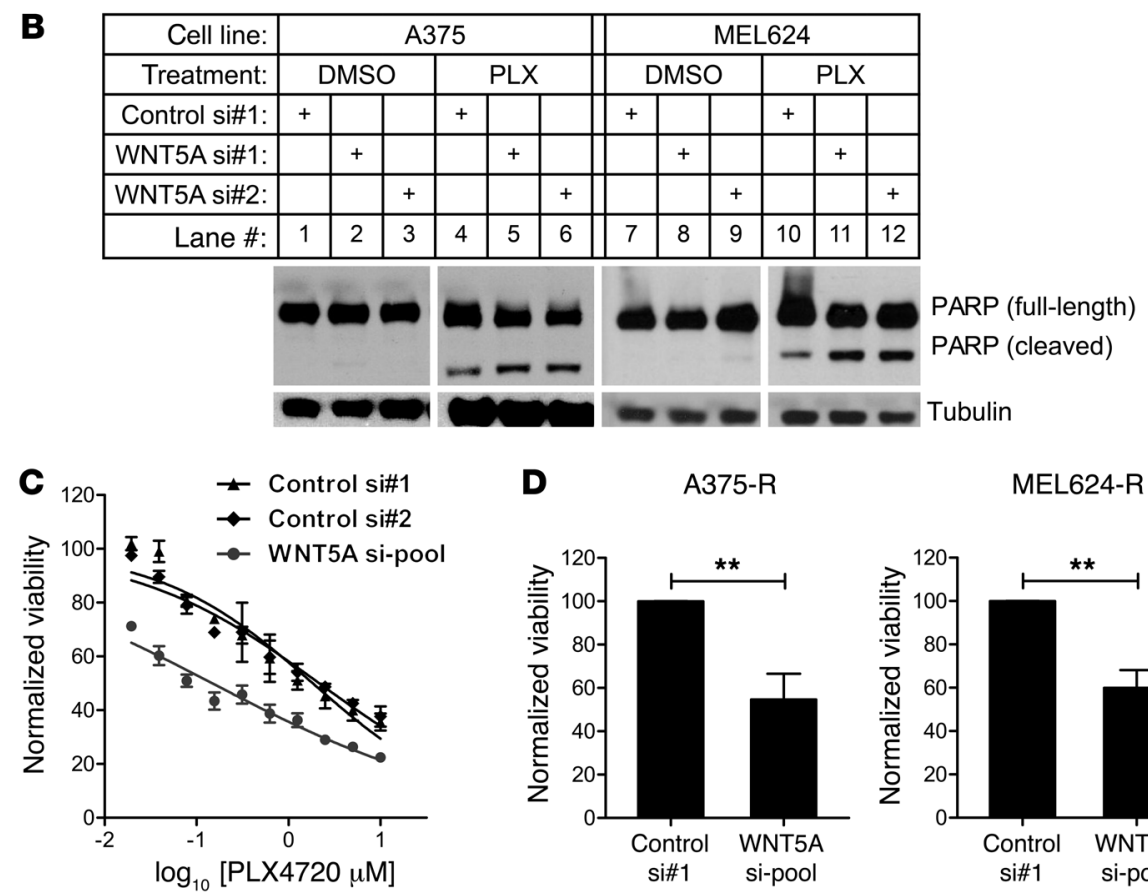

A375-R

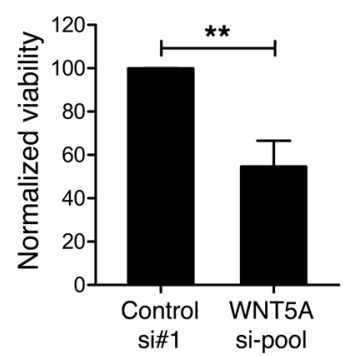

MEL624-R

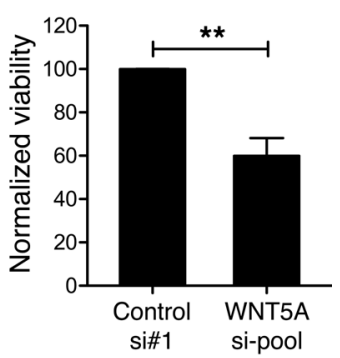

Figure 3

WNT5A loss of function decreases the viability of both PLX-naive and PLX-resistant melanoma cells. (A) Quantification of the percentage of A375 cells that were apoptotic (TUNEL+) after siRNA transfection and treatment with either DMSO or $2 \mu \mathrm{M}$ PLX4720 for 2 days. Error bars indicate the SD. ( ${ }^{*} P<0.05$, 2-tailed $t$ test.) (B) Western blot analysis of lysates from A375 (lanes 1-6) or MEL624 cells (lanes 7-12) transfected with the indicated siRNAs and then treated 2 days later with either DMSO (lanes 1-3, 7-9) or $2 \mu$ M PLX4720 (lanes 4-6, 10-12) for 48 hours. Blots were probed with either PARP antibody or $\beta$-tubulin antibody as a loading control. (C) Normalized viability of A375 cells after transfection with siRNAs followed by treatment with increasing doses of PLX4720 for 2 days. Normalized viability was determined with the mock + DMSO condition set at $100 \%$. Error bars show SD calculated from 3 independent experiments. Nonlinear best fit curves were obtained using GraphPad software (control siRNA \#1, $R^{2}=0.9472$; control siRNA \#2, $R^{2}=0.9144$; and WNT5A siRNA pool, $R^{2}=0.9016$ ). (D) Normalized viability of A375-R (left) and MEL624$\mathrm{R}$ (right) cells that were plated in medium containing $2 \mu \mathrm{M}$ PLX4720 48 hours after siRNA transfection and allowed to grow for 3 days. Data were normalized to the control siRNA \#1 condition set to $100 \%$, and error bars indicate SD of 3 independent experiments. $\left({ }^{\star \star} P<0.01,2\right.$-tailed $t$ test.)

such as SOX9, RUNX2, and TWIST2, extracellular matrix components such as COL5A1 and LAMC, and proteins involved in signal transduction such as VEGFC and EGFR (Figure 1D). These results suggest that increased WNT5A expression is induced in BRAFiresistant cell populations as part of a unique transcriptional program associated with drug resistance.

We observed increased WNT5A expression in both patient tumors that developed resistance to BRAF-targeted therapy and multiple cultured melanoma cell lines chronically treated with BRAFi in vitro. Our finding that WNT5A levels were increased in melanoma cells grown in vitro suggests that increased WNT5A observed in patient tumors is derived from the tumor tissue rather than other cells in the tumor microenvironment. This hypothesis is further supported by a recent study reporting increased expression of WNT5A by immunohistochemistry in the tumor cells in melanoma biopsies from patients showing a poor clinical response to BRAFi compared with patients who initially responded well to BRAFi (28).

WNT5A loss of function reduces melanoma cell growth. The observation that WNT5A expression was elevated in BRAFi-resistant melanomas raises the question of whether WNT5A functionally contributes to the growth and survival of melanoma cells. To address this question we determined whether knockdown of endogenous WNT5A regulates melanoma growth. For these studies we used 2 independent WNT5A siRNAs that consistently reduce levels of endogenous WNT5A protein and transcripts (Supplemental Figure 1, A and C). WNT5A knockdown inhibited the growth of both A375 and MEL624 cells to a similar degree to BRAF knockdown (Figure 2A). To test for the specificity of these siRNAs, we performed a rescue experiment by treating siRNA-transfected cells with WNT5A-conditioned medium. WNT5A-conditioned medium partially rescued growth inhibition induced by WNT5A knockdown (Supplemental Figure 1D). We then asked whether reducing endogenous WNT5A with siRNA also reduces melanoma growth in anchorage-independent growth assays (29). WNT5A siRNAs reduced colony formation in soft agar by more than $80 \%$ in both A375 and MEL624 cells and similarly reduce melanosphere growth (Figure 2, B-D).

WNT5A loss of function decreases the viability of BRAFi-resistant melanoma cells. We then determined whether WNT5A-dependent signaling also contributes to melanoma cell viability in the presence of BRAFi. siRNA knockdown of WNT5A enhanced the apoptotic response induced by short-term cotreatment with BRAFi, as indicated by a significantly increased proportion of cells labeled by TUNEL and by an increase in the abundance of cleaved PARP, an additional marker of apoptosis (Figure 3, A and B). Since a relatively small percentage $\left(\sim 10 \%-25 \% \mathrm{TUNEL}^{+}\right)$of melanoma cells underwent apoptosis under these conditions, we also tested whether combining WNT5A siRNAs with BRAFi can reduce cell 
viability as measured using resazurin, a metabolic indicator (see Methods). We found that pooled WNT5A siRNA reduced A375 cell viability at all doses of BRAFi (PLX4720) to $40 \%-50 \%$ of levels observed following mock transfection or transfection with control siRNAs (\#1 and \#2) (Figure 3C). As WNT5A expression was elevated in BRAFi-resistant melanoma cells (Figure 1), we then asked whether WNT5A is also necessary for the viability of the BRAFiresistant A375-R and MEL624-R cells. Similarly to the naive cell lines, transfection with pooled WNT5A siRNA decreased viability of both MEL624-R and A375-R cells when grown in PLX4720-containing medium (Figure 3D). As 2 independent WNT5A siRNAs similarly inhibited the growth of A375-R cells (see below), reduced A375-R and MEL624-R cell numbers observed following transfection with pooled WNT5A siRNAs are therefore not likely to be due to an off-target effect of a single siRNA. Collectively, these data suggest that WNT5A is required for optimal growth and survival of both naive and BRAFi-resistant melanoma cells.

Overexpression of WNT5A enhances melanoma cell growth. Since loss of endogenous WNT5A reduces the growth and viability of both naive and BRAFi-resistant melanoma cells, we next asked whether WNT5A overexpression is sufficient to promote melanoma growth. We infected melanoma cells expressing low levels of endogenous WNT5A (Supplemental Figure 1B) with lentiviruses to overexpress either GFP as a control or WNT5A and then monitored the expression of exogenous WNT5A by immunoblotting (Figure 4A). As we predicted, WNT5A overexpression promoted the growth of both A2058 and A375 cells in soft agar, as WNT5Aexpressing cells produced both an increased number of colonies compared with GFP control cells and larger colonies than control cells (Figure 4, B-D). Since the growth of cancer cells in soft agar involves the clonogenic expansion of individual cells, we hypothesized that WNT5A might promote the survival of single melanoma cells. To test this hypothesis, we plated single SK-MEL-28 melanoma cells in 96-well low-attachment plates and allowed these cells to grow undisturbed for 1 week. Approximately $30 \%$ of the single GFP control cells survived and proliferated to form colonies (Figure 4E), while a significantly higher percentage of the WNT5Aexpressing cells $(48 \%)$ formed colonies in this assay $(P<0.001)$.

Given that WNT5A overexpression enhanced the growth of melanoma cells in vitro, we then asked whether WNT5A can also promote tumorigenesis in vivo using a xenograft model. We transplanted 50,000 GFP- or WNT5A-transduced A2058 cells s.c. in the flanks of immunocompromised nude mice and then monitored the formation of tumors. Overexpression of WNT5A decreased the time to palpable tumor formation following cell transplantation (Figure 4F). Together, these results reveal that overexpression of WNT5A is sufficient to promote the growth of melanoma cells in vitro and enhances the tumorigenic potential of melanoma cells in vivo.

WNT5A negatively regulates the WNT/ $\beta$-catenin pathway in melanoma. In order to gain further insight into the observed role for WNT5A in promoting the viability of both naive and BRAFi-resistant melanomas, we then focused on identifying signaling pathways acting downstream of WNT5A in melanoma. We and others have recently shown that activation of WNT/ $\beta$-catenin-dependent signaling with WNT3A can inhibit the proliferation of melanoma cells in vitro and in vivo $(24,30,31)$. Since several studies indicate that WNT5A can act as a negative regulator of WNT/ $\beta$-catenin-dependent transcription $(32,33)$, these data suggest that WNT5A might promote melanoma growth by inhibiting WNT/ $\beta$-catenin signaling. We monitored WNT/ $\beta$-catenin signaling using a luciferase reporter of $\beta$-catenin-dependent transcription ("pBAR") (34) and found that WNT3A-conditioned medium but not WNT5A-conditioned medium enhanced $\beta$-catenin-dependent transcription in both A375 and A2058 melanoma cells (Supplemental Figure 2, A and B). As a control, we confirmed that activation of this luciferase reporter activity was due to increased activity of the $\beta$-catenin pathway by blocking reporter activation with a siRNA targeting CTNNB1 (encodes for $\beta$-catenin). Knocking down $\beta$-catenin effectively reduced $\mathrm{pBAR}$ reporter activity induced by WNT3A (Supplemental Figure 2C).

Since A375 cells endogenously expressed WNT5A, we then asked whether reducing WNT5A by siRNA transfection can either enhance or inhibit the activation of $\beta$-catenin-dependent transcription induced by WNT3A. In contrast to the CTNNB1 siRNA, knockdown of endogenous WNT5A with multiple individual siRNAs increased the sensitivity of A375 cells to stimulation with WNT3A-conditioned medium as indicated by a leftward curve shift (Supplemental Figure 2C). These data revealed that endogenous WNT5A acts as a negative regulator of the WNT/ $\beta$-catenin pathway in A375 cells. We then asked whether exogenous WNT5A can also negatively regulate $\beta$-catenin-dependent transcription. As we predicted, adding WNT5A-conditioned medium inhibited $\beta$-catenin-dependent transcription induced by WNT3A in multiple human melanoma cell lines (Supplemental Figure 2D). Consistent with previous studies involving other cell and tissue types $(32,35)$, these data suggest that WNT5A negatively regulates the WNT/ $\beta$-catenin pathway in melanoma.

Since WNT5A promoted melanoma growth and survival (Figures $2-4)$, whereas $\mathrm{WNT} / \beta$-catenin signaling inhibits melanoma growth and survival $(24,30,31)$, we then determined the consequences of simultaneously activating $\mathrm{WNT} / \beta$-catenin-dependent signaling with WNT3A-conditioned medium and inactivating WNT5A-dependent signaling by siRNA-mediated knockdown of WNT5A. Either knockdown of WNT5A with siRNAs or treatment with WNT3A-conditioned medium resulted in reduced A375 cell number (Supplemental Figure 3A). Cotreatment with WNT3Aconditioned medium and WNT5A siRNAs further reduced A375 cell proliferation compared with either treatment alone (Supplemental Figure 3A). These data revealed that a shift from elevated WNT/ $\beta$-catenin signaling to elevated WNT5A-dependent signaling can result in increased melanoma growth and survival.

Cross-talk between WNT/ $\beta$-catenin-dependent signaling and WNT5Adependent signaling does not account for decreased melanoma proliferation induced by WNT5A siRNAs. Based on previous reports suggesting that WNT5A can act as a negative regulator of WNT/ $\beta$-catenin signaling, we next tested our hypothesis that WNT5A loss of function might inhibit melanoma cell growth by promoting $\mathrm{WNT} / \beta$-catenin-dependent signaling. We determined the consequences of blocking the activation of WNT/ $\beta$-catenin-dependent transcription induced by WNT5A siRNA by treating cells with XAV939, a small-molecule inhibitor of WNT/ $\beta$-catenin signaling (36). XAV939 treatment efficiently blocked $\beta$-catenin-dependent transcription induced by WNT5A siRNAs (Supplemental Figure 3B). However, cotreatment with XAV939 and WNT5A siRNAs did not prevent the reduction in melanoma cell number induced by WNT5A loss of function (Supplemental Figure 3C). These results suggest that WNT5A acts as a negative regulator of the WNT/ $\beta$-catenin signaling pathway, yet increased activity of the WNT/ $\beta$-catenin signaling pathway does not cause the growth defects observed following WNT5A knockdown. 
A

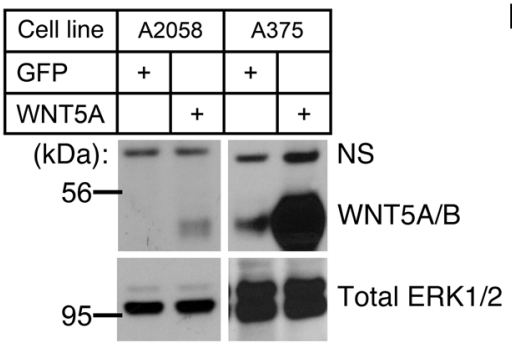

B

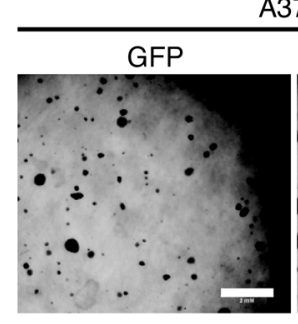

A375

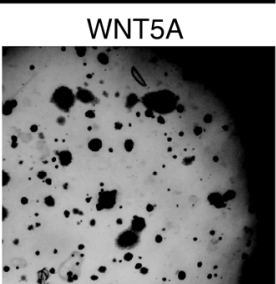

A2058

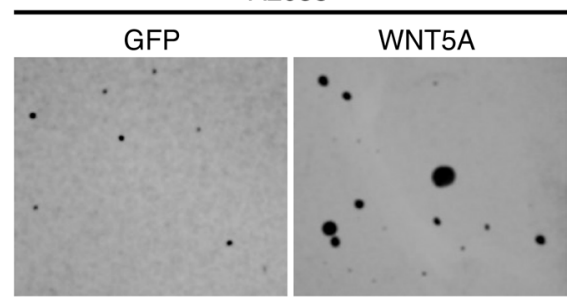

C

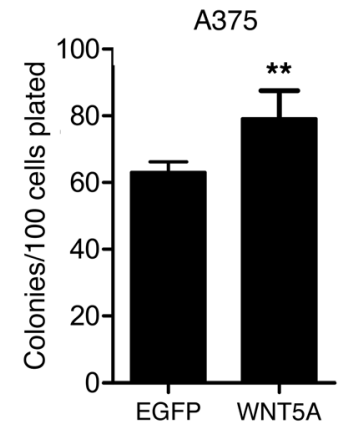

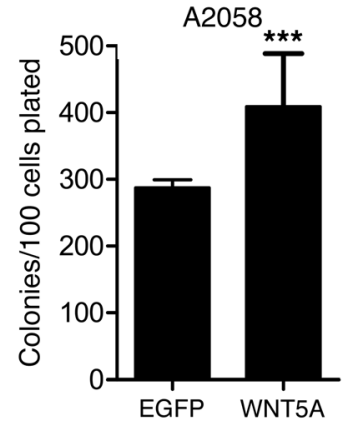

Day 10 melanospheres

E
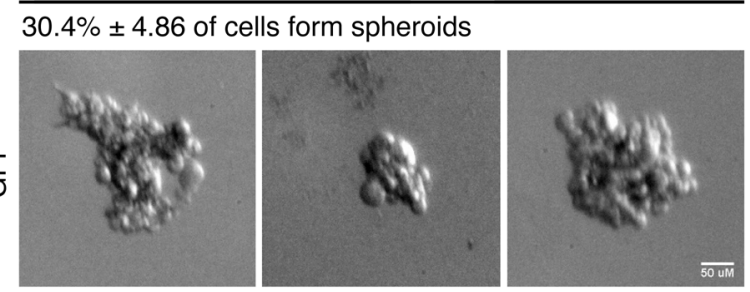

$48.3 \% \pm 6.3$ of cells form spheroids
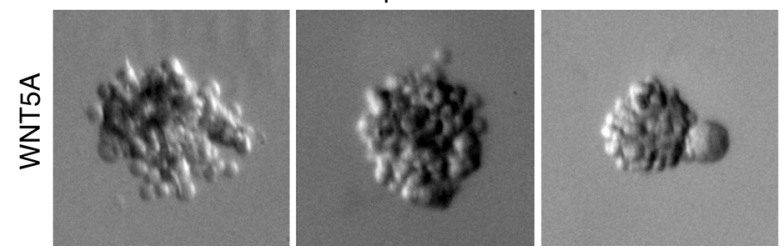

D

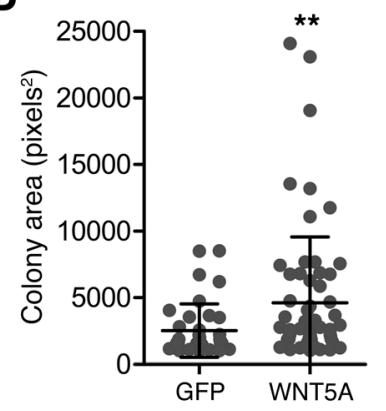

$\mathbf{F}$

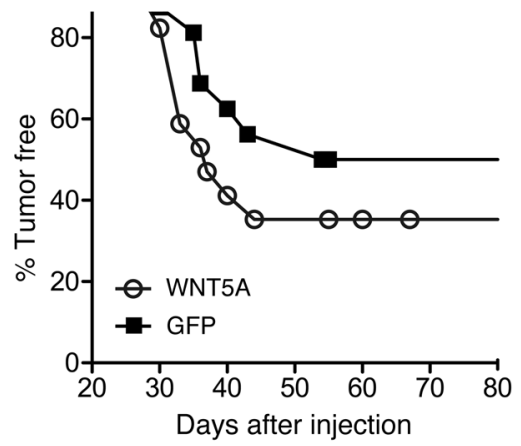

Figure 4

WNT5A overexpression enhances melanoma cell proliferation. (A) Representative Western blots of lysates collected from A2058 and A375 stable cell lines generated by infection with either WNT5A-IRES-GFP or IRES-GFP control lentiviruses. Blots were probed with antibodies to detect the expression of WNT5A and total ERK1/2. (B) Representative photographs of colonies formed 3 weeks after embedding of $100 \mathrm{~A} 375$ cells or 1,000 A2058 cells in soft agar. (C) Average number of colonies formed in anchorage-independent growth conditions per 100 A375 cells (left) or 1,000 A2058 cells (right) plated in soft agar. (D) Quantification of A2058 soft agar colony area. Each point represents the area of a single colony determined by image analysis. Center bars indicate the mean colony area, and error bars show the SD. Statistical significance of differences in colony number and melanosphere size was calculated using a 2-tailed Student's $t$ test. ( ${ }^{\star \star} P<0.01,{ }^{\star * \star} P<0.001$.) (E) Summary of the spheroid-forming potential of SK-MEL-28 cells infected with either WNT5A or GFP control lentiviral particles. Representative photographs of melanospheres taken 10 days after plating of single cells are included below the table summarizing the proportion of sphere-forming cells compiled from 3 independent experiments. (F) Kaplan-Meier survival curve indicating the tumor-free survival of mice injected with 50,000 A2058 melanoma cells expressing either WNT5A or GFP controls.

WNT5A enhances AKT pathway activity in melanoma. Since WNT5A did not appear to promote melanoma growth and survival by acting as an inhibitor of WNT/ $\beta$-catenin signaling, we then focused on identifying other signaling pathways that might be modulated by WNT5A in melanoma. We next determined whether WNT5A enhances melanoma cell growth via kinasedependent signaling pathways. For these experiments, we monitored the phosphorylation of different kinases in A375 cells by transfecting the cells with either control or WNT5A siRNAs and then subjecting them to a short time course of serum stimulation (Figure 5A). As a positive control, we examined the phosphorylation of PKC, which is normally induced by WNT5A in melanoma $(37,38)$. As expected, transfection of A375 cells with both pooled WNT5A siRNAs and individual WNT5A siRNAs \#1 and $\# 2$ reduced the phosphorylation of $\mathrm{PKC}$ in comparison with control siRNA (Figure 5A). 
A

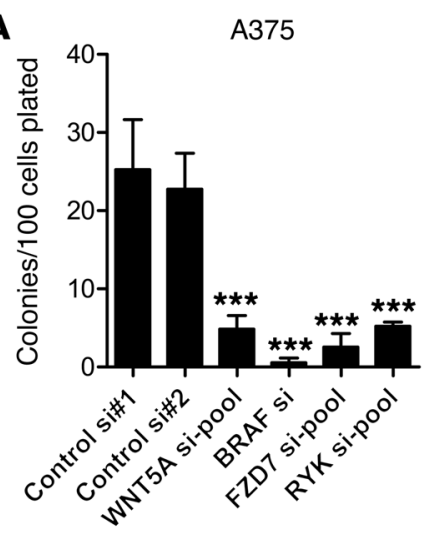

C

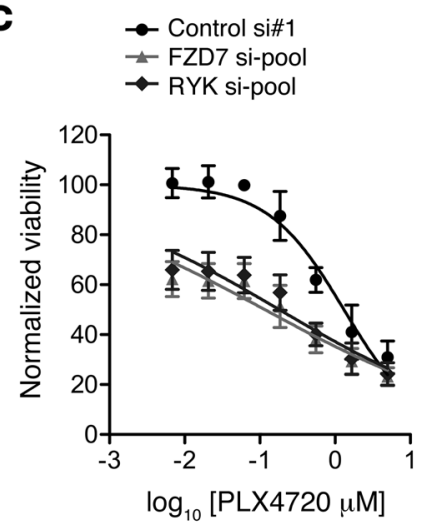

MEL624
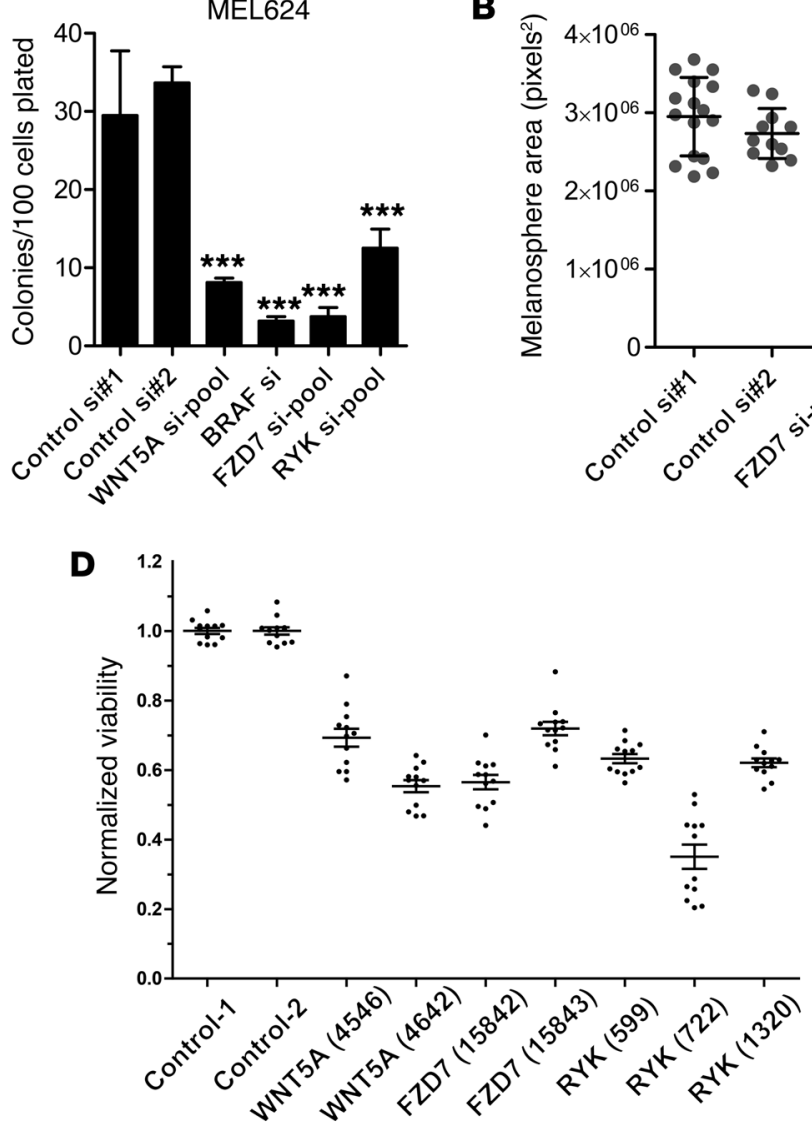

B

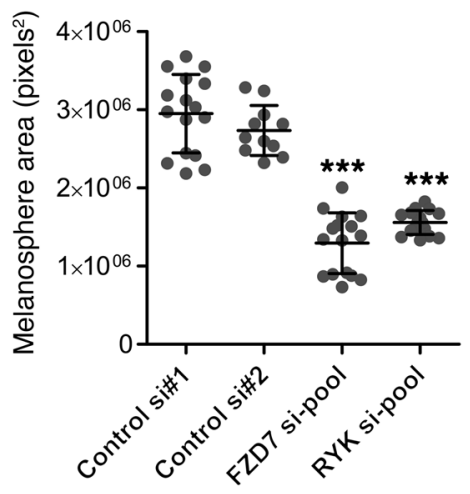

E

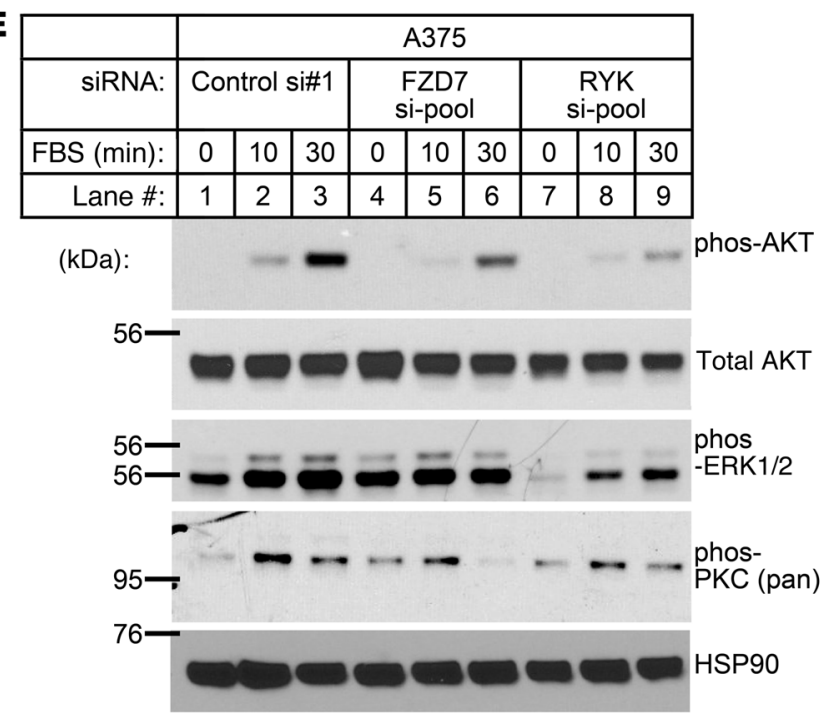

\section{Figure 6}

The WNT5A receptors FZD7 and RYK promote melanoma cell growth and AKT phosphorylation. (A) Average number of colonies formed in soft agar after transfection of A375 (left) and MEL624 (right) cells with pooled siRNAs followed by culture in soft agar for 1 week. (B) Quantification of A375 melanosphere area. Statistical significance of differences in soft agar colony number and in melanosphere size was calculated by ANOVA. $\left({ }^{* *} P<0.01\right.$, ${ }^{* *} P<0.001$.) (C) Normalized viability of A375 cells that were transfected with the indicated siRNAs, then subsequently treated with increasing concentrations of PLX4720 2 days later. Transfected cells were grown in the presence of PLX4720 for a total of 3 days before viability was determined. Values were normalized to control siRNA with DMSO set at $100 \%$, and nonlinear, best fit regression curves were generated using GraphPad. (D) Average cell viability of $A 375$ cells plated at a density of 5,000 cells per well in 96 -well plates 2 days after transfection with either control siRNAs or siRNAs targeting WNT5A, RYK, and FZD7. Cells were then allowed to grow for 3 days, and cell viability was determined. All siRNAs are $P<0.0001$ by 1 -way ANOVA and Dunnett's post-test comparison with control columns. (E) Western blots of lysates from A375 cells transfected with either control siRNA \#1 (lanes 1-3), pooled FZD7 siRNAs (lanes 4-6), or pooled RYK siRNAs (lanes 7-9). Blots were probed with antibodies to detect the phosphorylated forms of ERK1/2 (Thr202, Tyr204), AKT (Ser473), and PKC (pan-phospho- $\beta$ II Ser660) and HSP90 as a loading control.

These data were consistent with previous reports of hyperactivated PI3K/AKT signaling in BRAFi-resistant melanomas $(16,43,44)$.

Several studies have reported that cotreating melanoma cells with BRAF/MAPK and PI3K/AKT inhibitors synergistically reduces cell viability $(41,42,44-47)$, suggesting that the BRAF/MAPK and PI3K/AKT pathways cooperate to promote cell growth. We 
similarly found that cotreating naive melanoma cells with an $\mathrm{IC}_{50}$ dose of PLX4720 and with increasing doses of either GDC-0941 (a PI3K inhibitor) or BEZ235 (a dual PI3K/mTOR inhibitor) further reduced cell viability compared with treatment with BRAFi alone (Supplemental Figure 7, A and B). As a control, we then asked whether our BRAFi-resistant cell lines also require PI3K/ AKT pathway activity for their growth and found that GDC-0941 and BEZ235 also reduced the viability of A375-R and MEL624-R cells (Supplemental Figure 7, C and D). In summary, these results revealed that WNT5A can activate $\mathrm{PI} 3 \mathrm{~K} / \mathrm{AKT}$ signaling that is required for the optimal viability of both naive and BRAFi-resistant cells. Our findings are consistent with the hypothesis that WNT5A enhances the growth and survival of melanoma cells by activating $\mathrm{PI} 3 \mathrm{~K} / \mathrm{AKT}$ signaling.

The WNT5A receptors FZD7 and RYK are highly expressed in melanoma. Previous work has identified multiple putative WNT5A receptors that act in a cell- and tissue type-dependent manner $(48,49)$. Since previous studies indicate that WNT5A is highly expressed in metastatic melanomas that display poor clinical outcomes $(38,50-52)$, we reasoned that functional WNT5A receptors would also be highly expressed in melanomas. We analyzed a large microarray data set profiling transcript expression in 950 human tissue samples including multiple normal and cancerous tissues (ArrayExpress, E-MTAB-37). We found that FZD6 $\left(P=4.93 \times 10^{-5}\right)$, FZD7 (probe $1, P=3.84 \times 10^{-9}$; probe 2, $P=4.94 \times 10^{-10}$ ), and $R Y K$ (probe $1, P=4.76 \times 10^{-4}$; probe 2 , $P=2.80 \times 10$; probe $\left.3, P=1.45 \times 10^{-6}\right)$ were significantly elevated in melanoma while other WNT receptors were not (Supplemental Figure 8A). We analyzed additional microarray data sets and found that FZD7 was highly expressed in both normal skin and melanoma (Supplemental Figure 4B), while RYK was overexpressed in melanoma (Supplemental Figure 8B).

siRNAs targeting the WNT receptors FZD4, FZD5, FZD7, and RYK reduce melanoma cell viability and growth. In parallel to our analysis of gene expression profiles, we also carried out functional tests to determine whether any of these WNT receptors contributed to melanoma cell viability in a manner similar to that of WNT5A. As expected from previous results, we found that BRAF and WNT5A siRNAs reduced A375 cell viability, whereas transfection with either control siRNAs or siRNAs targeting LRP6 (Supplemental Figure $9 \mathrm{H}$ ) and $\beta$-catenin (24) to block WNT/ $\beta$-catenin signaling had no effect on cell viability (Supplemental Figure 10A). Previous reports have suggested that the receptor tyrosine kinases ROR1 and ROR2 can act as WNT5A receptors and that they enhance the proliferation of cancer cells $(28,53,54)$. We observed efficient knockdown of ROR1 and ROR2 transcripts with all 3 siRNAs (Supplemental Figure 9, F and G), yet cell viability decreased with only 1 of 3 ROR 1 and 1 of 3 ROR2 siRNAs (Supplemental Figure 10A). These results suggest that an off-target effect of a single siRNA rather than variable knockdown of ROR1 and ROR2 by different siRNAs probably accounts for the lack of consistent changes in cell number observed following ROR1 or ROR2 depletion. In contrast to ROR1 and ROR2, we found that multiple validated siRNAs targeting the WNT5A receptor RYK (55) inhibited the growth of A375 melanoma cells (Supplemental Figure 10A).

We also tested whether members of the Frizzled family of receptors found to be highly expressed in melanoma (Supplemental Figure 8A) also regulate the growth of melanoma cells in vitro, by transfecting A375 cells with additional siRNAs targeting FZD1, FZD4, FZD5, FZD6, and FZD7. We first confirmed that these siRNAs effectively reduce their target transcripts by qRTPCR, and found that all siRNAs effectively reduced their target mRNAs (Supplemental Figure 9, A-E). We did not consistently observe a statistically significant change in cell viability following transfection with siRNAs targeting FZD1 or FZD6 (Supplemental Figure 10B), as only 1 of 2 FZD 1 siRNAs and 1 of 2 FZD 6 siRNAs significantly reduced A375 cell growth in this assay. We did observe a reduction in cell viability after transfection with multiple, independent FZD7-, FZD4-, and FZD5-targeted siRNAs (Supplemental Figure 10B).

Since FZD4 and FZD5 were expressed at very low levels in the melanoma cells used in this study (Supplemental Figure 9, A and B), we decided to focus our further studies on RYK and FZD. We further validated that multiple siRNAs targeting both RYK and FZD7 reduced melanoma cell number by manually counting A375 cells following transfection with these siRNAs and observed that multiple, independent siRNAs targeting these receptors decreased cell number (Supplemental Figure 10C). Since loss of WNT5A inhibited melanoma proliferation in 3D culture assays (Figure 2, B-D), we asked whether loss of FZD7 and RYK receptors similarly regulates melanoma cell growth in these assays. Like depletion of WNT5A, depletion of either FZD7 or RYK by transfection with pooled siRNAs inhibited the growth of both A375 and MEL624 melanoma cells when grown in soft agar assays and when grown as melanospheres embedded in Collagen I (Figure 6, A and B).

We then determined whether RYK and FZD7 also regulate melanoma cell growth in the presence of BRAFi. We again treated melanoma cells with increasing concentrations of PLX4720 following transfection with pooled RYK and pooled FZD7 siRNAs. Similar to our findings with reduced WNT5A levels (Figure 3), siRNAmediated reduction of either RYK or FZD7 further reduced melanoma cell growth at all doses of PLX4720 (Figure 6C). Finally, we asked whether FZD7 and RYK are also required for the growth and survival of BRAFi-resistant melanoma cells. Similarly to the naive cell lines, siRNA knockdown of either RYK or FZD7 using pooled siRNAs decreased the growth of both A375-R and MEL624-R cells, as did knockdown of these receptors using multiple independent siRNAs (Figure 6D and Supplemental Figure 11). Taken together, these results support the hypothesis that WNT5A enhances the growth and viability of both naive and BRAFi-resistant melanoma cells via the receptors RYK and FZD7.

FZD7 and RYK enhance AKT signaling in melanoma. As our data demonstrated that elevating levels of WNT5A enhanced AKT signaling in melanoma, we predicted that increased expression of receptors for WNT5A, such as FZD7 and RYK, would also regulate AKT signaling in melanoma. Transfection of A375 cells with ssFLAG-FZD7 enhanced AKT phosphorylation, but not ERK phosphorylation, in comparison with FLAG-GFP (Supplemental Figure 12). Overexpression of RYK had little effect. We also investigated whether endogenous FZD7 and RYK can regulate the phosphorylation of AKT in melanoma cells. As with WNT5A siRNAs, transfecting A375 cells with either pooled RYK or pooled FZD7 siRNAs reduced AKT phosphorylation induced by serum stimulation, but did not significantly affect either ERK1/2 or PKC phosphorylation (Figure 6E).

WNT5A is upregulated as part of a gene signature associated with clinical phenotypes in melanoma. Our microarray analysis suggested that WNT5A expression is elevated in concert with a group of other transcripts in BRAFi-resistant versus naive melanoma cell lines (Figure 1D). Since WNT5A loss and gain of function regulated 


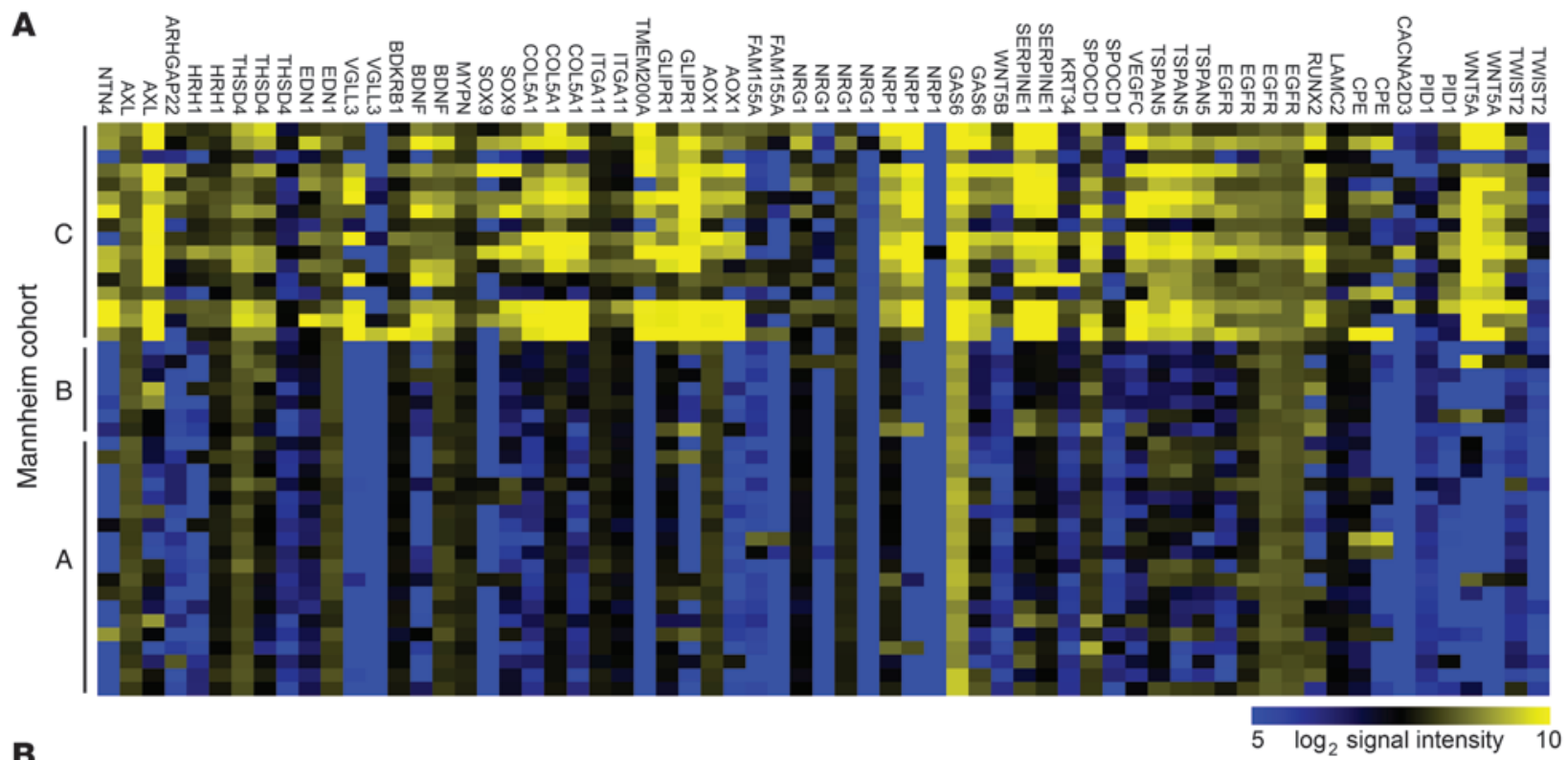

B

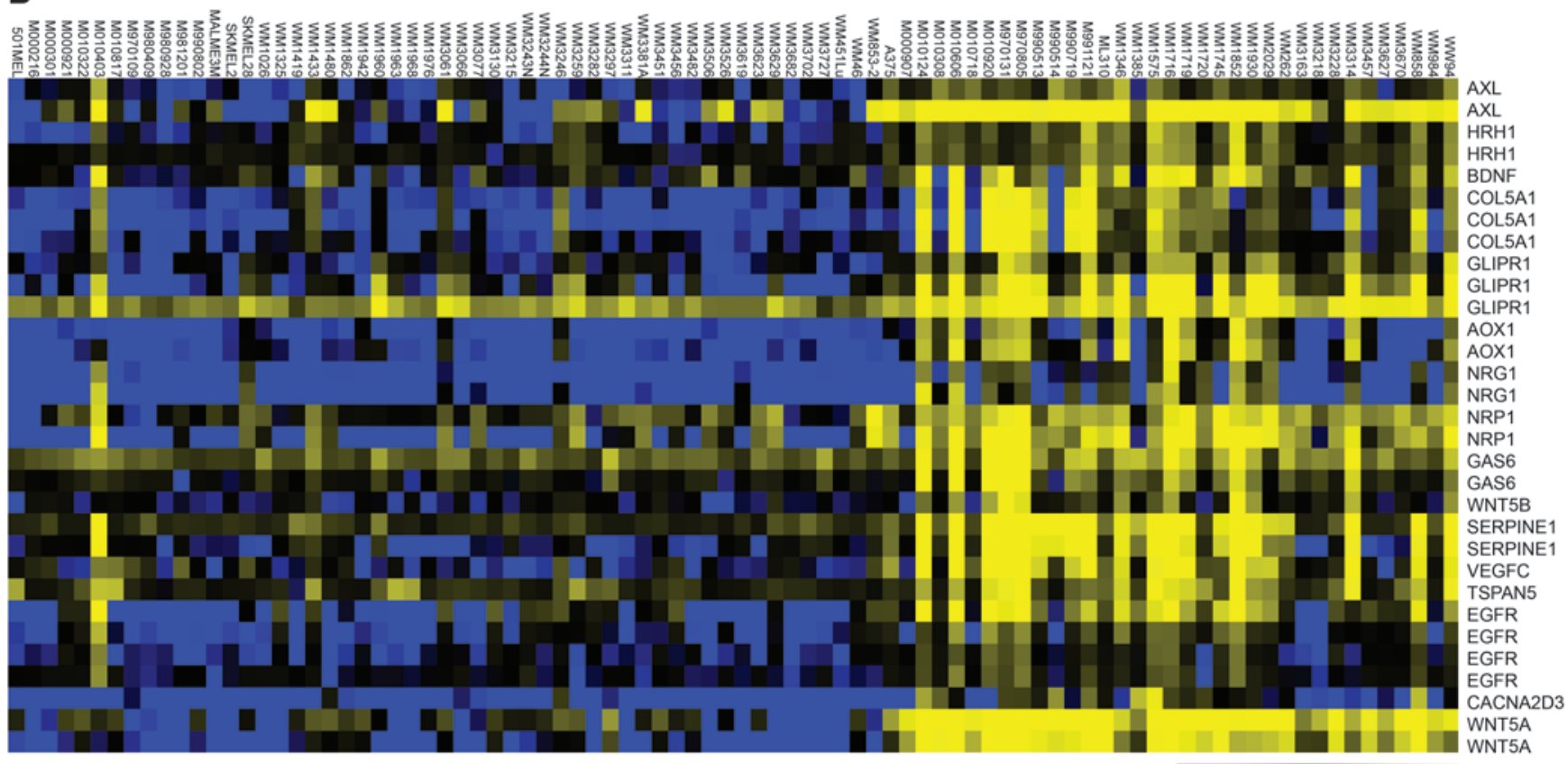

$5 \log _{2}$ signal intensity 10

\section{Figure 7}

WNT5A is upregulated as part of a gene signature associated with clinical phenotypes in melanoma. (A) The gene set upregulated in conjunction with WNT5A in BRAFi-resistant melanoma cells from Figure 1D was assessed in the previously published Mannheim data set, in which melanoma samples were clustered on the basis of either a highly metastatic phenotype (cohort $C$ ) or a less metastatic phenotype (cohorts $A$ and $B$ ). The gene probes in the data set that were significant by ANOVA analysis $(P<0.01)$ are displayed in this heat map. Signal intensities are shown as $\log _{2}$-transformed data. (B) The same gene set from Figure 1D was assessed in the Broad Institute's database of 88 melanoma samples (cell lines and short-term cultures). The heat map shows probes from the gene set that are significantly different $(t$ test, $P<0.01)$ between samples with low and high expression of WNT5A.

melanoma cell growth survival (Figures 3 And 4), we asked whether this gene expression signature is able to distinguish between different clinical subtypes of melanoma tumors. Indeed, in 1 data set, genes that we correlated with WNT5A overexpression in longterm BRAFi-treated cells were significantly enriched in a subset of patient samples characterized by high metastatic potential $(P<0.01$, ANOVA; Figure $7 \mathrm{~A}$ and ref. 56). This gene signature was also upregulated in a subset of melanoma cell lines and shortterm primary cultures of melanomas from an independent study (Figure 7B and ref. 57). These data suggest that the genes elevated 
concurrently with WNT5A in BRAFi-resistant melanomas (Figure 1D) also correlate with high WNT5A expression in other melanoma cell data sets independently of BRAFi treatment. Together, these data suggest that cells with higher levels of WNT5A expression may represent a distinct cellular phenotype that can be either induced or enriched by long-term BRAFi treatment.

\section{Discussion}

A more complete understanding of melanoma progression and responses to targeted therapeutics such as BRAFis will be crucial for developing more durable therapies. In this study, we found that WNT5A was dramatically upregulated in a subset of BRAFi-resistant melanoma tumors and in BRAFi-resistant melanoma cells derived in vitro. We further showed that WNT5A promoted the growth and survival of both naive parental cells and BRAFi-resistant melanoma cells. Previous reports found that high expression of WNT5A similarly results in reduced responsiveness of colon cancer cells to histone deacetylase inhibitors (21) and reduced responsiveness of pancreatic cancer cells to gemcitabine (58). Along with these previous studies, our current work highlights an emerging role for WNT5A-dependent signaling in mediating drug resistance in cancer.

Resistance to the targeted inhibition of the BRAF/MAPK pathway in melanoma involves perturbations in multiple different signaling axes. BRAFi-resistant melanoma cells often exhibit crossresistance to other BRAF/MAPK pathway inhibitors such as drugs targeting MEK $(44,59,60)$, suggesting that aberrant activity of other signaling pathways and cellular processes can compensate for the loss of BRAF/MAPK signaling. Indeed, many studies have observed increased activity of key mitogenic signaling proteins such as RTKs and AKT in BRAFi-resistant melanomas $(9,14,15$, 47), while other studies report that BRAFi-resistant melanoma cells inactivate apoptotic pathways through downregulation of $B I M(16)$ and upregulation of $M D M 4$, a negative regulator of TP53 (18). In addition to these cell-autonomous mechanisms of resistance to RAF inhibitors, recent studies suggest an important role for secreted proteins such as HGF in the development of BRAFi resistance $(61,62)$. The results of the present study add WNT5A to this growing list of secreted signaling proteins that may promote both intrinsic and acquired resistance to BRAF/MEK inhibition.

Specific interest in the role of WNT5A in melanoma dates back to transcriptional profiling studies that observed elevated levels of WNT5A in metastatic melanoma (50). Subsequent immunohistochemistry studies showed that high expression of cytoplasmic WNT5A correlates with disease progression, decreased progression-free survival, and decreased overall survival (51). Further mechanistic studies focused largely on the role of WNT5A in regulating cell motility through the ROR2/PKC pathway as well as through regulating the polarization of cell adhesion molecules (38, $39,63)$. Our results suggested that WNT5A can activate multiple signaling pathways in melanoma cells, since we found that WNT5A and its receptors FZD7 and RYK all promoted AKT activation. During the course of our studies, another group similarly observed that FZD7 can activate AKT in muscle progenitor cells (64), suggesting that WNT/FZD7/AKT signaling is active in multiple cell types. Although we cannot rule out the possibility that other WNT receptors also contribute to WNT5A-dependent cell proliferation and survival in melanoma, these studies represent an initial characterization of a WNT5A/AKT pathway that may be therapeutically relevant in metastatic and drug-resistant melanomas.
Our detailed analysis of gene expression in BRAFi-resistant melanoma cells revealed that WNT5A was not upregulated in isolation, but rather in concert with numerous other genes as part of a unique gene expression signature. This gene expression signature was also coordinately upregulated in metastatic subsets of melanomas even in the absence of BRAF or MAPK inhibitors $(56,57)$, which suggests that this gene signature identifies a phenotypic subtype of melanomas that can arise independently of therapeutic intervention. Future studies will address whether the genes upregulated in concert with WNT5A in both BRAFi-resistant and metastatic melanomas might collaborate with WNT5A in a unified signaling network to promote disease progression and drug resistance. Importantly, we found that WNT5A was upregulated not only in BRAFi-resistant cells derived in cell culture, but also in patients who have relapsed on BRAF/MAPK inhibitors. While we did not observe a significant correlation between WNT5A expression and survival in the small cohort of 11 patients analyzed in this study, further analysis of larger numbers of patient-derived BRAFi-resistant tumors may reveal whether WNT5A expression can predict clinical outcomes with better statistical power. Future studies will also determine whether the gene signature upregulated in concert with WNT5A in metastatic and BRAFi-resistant melanomas can more accurately predict clinical responses to BRAF/MAPK inhibition.

On the basis of our analysis of WNT5A-dependent signal transduction in cultured melanoma cells, we speculate that high WNT5A expression in patient tumors might similarly lead to increased PI3K/AKT activity. It will be interesting to determine whether WNT5A expression can serve as a biomarker to predict patient response to the PI3K and AKT inhibitors that are the subject of investigation as therapeutics for metastatic melanoma in ongoing clinical trials. Although it is still challenging to target WNT5A signaling pharmacologically, our results suggest that inhibiting this WNT5A/RYK/FZD7 pathway might be therapeutically beneficial in melanoma patients who have developed resistance to BRAF/MAPKtargeted therapies. FZD7 has recently garnered attention for its potential connection to the development and progression of multiple cancer subtypes (65). Blocking FZD7 activity with siRNAs or peptides can induce apoptosis and inhibit proliferation in vitro and in vivo in colon, breast, pancreatic, lung, and hepatocellular cancer cells (65-69). Whether these methods of blocking FZD7 activity could be adapted for the treatment of melanomas or other cancers exhibiting chemotherapeutic resistance due to aberrant hyperactivation of WNT5A-dependent signaling remains to be explored.

\section{Methods}

Analysis of WNT5A expression in melanoma patient samples. Access to patients undergoing early-phase clinical trials with vemurafenib and dabrafenib enabled us to acquire a unique set of triplicate melanoma biopsies before treatment and upon disease progression to study biomarkers of response and resistance to these drugs. Patients with BRAF-mutant metastatic melanoma with at least 2 lesions amenable to excision biopsy before and during systemic therapy with a BRAFi were included in this study. Informed consent was obtained for each patient under approved Human Ethics Review Committee protocols. Excision biopsies were taken within 7 days prior to commencement of the BRAFi (pretreatment samples). Biopsies of new or growing melanoma metastases (progressed or resistant samples) were performed in a subset of patients. The BRAFi was ceased no more than 24 hours before surgery for those requiring a general anesthetic for therapeutic surgical resection of progressed lesions. Patient samples were used to isolate mRNA, which was subsequently converted to cDNA and then 
analyzed for levels of WNT5A using SYBR Green real-time qRT-PCR. The team performing measurements of WNTSA transcript was blinded to the clinical outcome of patients. Statistical analysis for patient outcomes data was performed using IBM SPSS version 21 (SPSS).

Cell culture. Melanoma cell lines used in this study were cultured in various growth media purchased from Life Technologies supplemented with penicillin/streptomycin and FBS. Briefly, A375 cells were cultured in DMEM + 5\% FBS, A2058 cells were cultured in DMEM + 10\% FBS, SK-MEL-28 cells were grown in MEM + 10\% FBS, and COLO829, MEL624, M288, M238, and M14 cells were all cultured in RPMI + 10\% FBS. A375 and MEL624 BRAFi-resistant cell lines were generated by growing of these cells in $2 \mu \mathrm{M}$ PLX4720 (a BRAFi) for at least 10 weeks. During this process, cells were split once they reached confluence, and PLX4720-containing medium was replenished every 3-4 days. In order to generate additional resistant cell lines, COLO829, SK-MEL-28, M288, M238, and M14 were treated at their growth-adjusted inhibitory concentration of $50 \%\left(\mathrm{IC}_{50}\right)$ of PLX4032 (another BRAFi), or with DMSO as a control. Concentrations of PLX4032 were increased as resistance developed.

siRNA transfection. Melanoma cells were reverse-transfected with 10-20 nM siRNA (final concentration) in 6-well plates with RNAiMAX reagent per the manufacturer's suggestions (Life Technologies). The cata$\log$ numbers corresponding to the siRNAs used in this study can be found in Supplemental Table 1.

Generation of stable cell lines. A2058, A375, and UACC1273 melanoma cell lines expressing either IRES-GFP or WNT5A-IRES-GFP were generated by lentiviral transfection followed by FACS to isolate $\mathrm{GFP}^{+}$cells (30). SKMEL-28 cells expressing either GFP or WNT5A were generated by infection of these cells with additional lentivirus constructs to express either GFP or WNT5A followed by selection with hygromycin.

Analysis of $W N T / \beta$-catenin signaling. Production of WNT3A- and WNT5Aconditioned medium as well as assays of WNT/ $\beta$-catenin-dependent transcriptional activation were conducted using a $\beta$-catenin-activated luciferase reporter as previously described (34).

Cell proliferation and viability assays. Melanoma cells were trypsinized and counted 24-48 hours after transfection of the indicated siRNAs and then replated for various growth assays. To assess 2-dimensional growth, 50,000 A375 or MEL624 cells were plated in multiple wells of a 24-well plate 24 hours after siRNA transfection. At various time points following initial platting, cells were trypsinized and counted using a hemocytometer. All growth curves summarize 3 independent experiments performed with triplicate technical replicates for each time point and condition. For rescue experiments, cells were plated in either medium containing $25 \%$ control L cells, or $25 \%$ WNT5A-conditioned medium prepared as previously described. For resazurin-based viability assays, 2,500 to 5,000 cells were plated in 96-well plates 48 hours after siRNA transfection in normal growth medium and allowed to grow for $48-72$ hours. At this time, the normal cell culture medium was exchanged with medium containing resazurin (Promega), and plates were incubated for 2.5-3 hours in the tissue culture incubator before assessment of the fluorescent end product (resorufin) using an EnVision plate reader (PerkinElmer).

Three-dimensional cell culture. Soft agar assays were performed by embedding of 100-1,000 siRNA-transfected cells at single-cell density in $0.3 \%$ agarose overlaid on a $0.6 \%$ base agarose layer in 6 -well plates. Macroscopic colonies were scored and colony-forming efficiencies were calculated 6-7 days after single cells were embedded in soft agar. Melanosphere assays were performed as previously described with minor modifications (29). Briefly, 24 hours after transfection of melanoma cells with various siRNAs, 5,000 cells were plated in multiple wells of ultralow-attachment roundbottom 96-well plates (Corning), where they grew into melanospheres. Melanospheres were embedded in Collagen I (BD Biosciences) 48 hours later and imaged 24 hours after embedding. Melanosphere areas were determined using Image (NIH) to measure the diameter of each sphere from micrographs. All measurements were done blinded to avoid potential experimenter bias.

In vivo tumorigenesis. Fifty thousand A2058 cells expressing either GFP or WNT5A-IRES-GFP were injected s.c. into the flanks of nude Fox $\mathrm{N1}^{-/-}$mice (Harlan Laboratories). Mice were checked 3 times a week and the presence of palpable tumors was determined. Tumor volumes were calculated using caliper measurements as previously described (70).

Western blotting. Cells were washed with ice-cold PBS ( $\mathrm{pH}$ 7.5), and then lysed before quantification of protein by bicinchoninic acid assay to equalize samples for Western blotting. Combinations of the following antibodies were then used to detect proteins of interest: anti-FLAG (clone M2, Sigma-Aldrich), anti-WNT5A/B (9562, Cell Signaling), anti-phospho-AKT (Ser473) (Cell Signaling), anti-total AKT (Cell Signaling), anti-phosphoERK1/2 (Cell Signaling), anti-total ERK1/2 (Cell Signaling), anti-phospho-JNK (Cell Signaling), anti-pan-phospho-PKC (Cell Signaling), antiphospho-PKC $\alpha / \beta$ (Cell Signaling), anti-PARP1 (Cell Signaling), anti-total GSK3 $\beta$ (Cell Signaling), anti-phospho-GSK3 $\beta$ (Millipore), anti-HSP90 (Santa Cruz Biotechnology), and anti- $\beta$-tubulin (Millipore). See complete unedited blots in the supplemental material.

Analysis of gene expression by microarray. For microarray analysis of gene expression, the RNA was isolated using the QIAGEN RNeasy protocol and quantitated using a NanoDrop Spectrophotometer (Agilent Technologies). Seven hundred fifty nanograms of high-quality total RNA with RIN (RNA integrity number) greater than 8.0 was labeled with cyanine 5-CTP or cyanine 3-CTP using the Low RNA Input Fluorescent Linear Amplification Kit (Agilent Technologies) and purified on RNeasy Mini columns (QIAGEN). Labeled RNA was then hybridized to Agilent Human 44K expression arrays that include 44,000 probes and compared with a labeled mixed reference sample consisting of a pool of equal amounts of RNA from each of 47 melanoma cell lines. The analysis of the microarray data was done using Rosetta Biosoftware's Resolver system. ROAST analysis was used to perform similarity searches to determine the correlation between WNT5A expression and other probes detecting transcripts with similar expression patterns. Raw and normalized array data have been uploaded to Gene Expression Omnibus at NCBI (GSE55583). All RNA isolation and expression microarray experiments were performed in triplicate.

Analysis of gene expression by RT-PCR. RNA was purified with the RNeasy kit according to the manufacturer's protocol (QIAGEN). cDNA was synthesized with RevertAid M-MuLV Reverse Transcriptase (Fermentas). LightCycler FastStart DNA Master SYBR Green 1 (Roche) was used for real-time PCR using various primers (Supplemental Table 2) to detect human mRNA transcripts.

Statistics. Statistically significant differences between data were determined using GraphPad software. One-way ANOVA or 2-tailed $t$ tests as indicated in the figure legends were used to compare most data sets. Microarray data sets were compared using 1-way ANOVA test for statistical significance. Unless otherwise indicated, all error bars indicate SD. $P$ values less than 0.05 were considered significant.

Study approval. All experiments involving patients and analysis of patient samples were carried out using protocols approved by the Royal Prince Alfred Hospital Ethics Review Committee under Protocol X10-0305 (HREC/10/RPAH/539). All experiments involving animals were approved by the IACUC at the University of Washington.

\section{Acknowledgments}

We would like to thank our colleagues in the Moon laboratory for their helpful comments. R.T. Moon is an investigator 
of the Howard Hughes Medical Institute. A.J. Chien and R.M. Kulikauskas are supported through bridge funding from the University of Washington Provost's Office. H. Rizos is a recipient of a Cancer Institute New South Wales Research Fellowship and a National Health and Medical Research Council Senior Research Fellowship.

Received for publication April 15, 2013, and accepted in revised form March 27, 2014.

1. Davies $\mathrm{H}$, et al. Mutations of the BRAF gene in human cancer. Nature. 2002;417(6892):949-954.

2. Curtin JA, et al. Distinct sets of genetic alterations in melanoma. NEnglJ Med. 2005;353(20):2135-2147.

3. Tsai J, et al. Discovery of a selective inhibitor of oncogenic B-Raf kinase with potent antimelanoma activity. Proc Natl Acad Sci U S A. 2008;105(8):3041-3046.

4. Joseph EW, et al. The RAF inhibitor PLX4032 inhibits ERK signaling and tumor cell proliferation in a V600E BRAF-selective manner. Proc Natl Acad Sci U S A. 2010;107(33):14903-14908.

5. Flaherty KT, et al. Inhibition of mutated, activated BRAF in metastatic melanoma. $N$ Engl J Med. 2010;363(9):809-819.

6. Sosman JA, et al. Survival in BRAF V600-mutant advanced melanoma treated with vemurafenib. NEngl J Med. 2012;366(8):707-714.

7. Bollag $\mathrm{G}$, et al. Clinical efficacy of a RAF inhibitor needs broad target blockade in BRAF-mutant melanoma. Nature. 2010;467(7315):596-599.

8. Chapman $\mathrm{PB}$, et al. Improved survival with vemurafenib in melanoma with BRAF V600E mutation. NEngl J Med. 2011;364(26):2507-2516.

9. Nazarian R, et al. Melanomas acquire resistance to B-RAF(V600E) inhibition by RTK or N-RAS upregulation. Nature. 2010;468(7326):973-977.

10. Wagle N, et al. Dissecting therapeutic resistance to RAF inhibition in melanoma by tumor genomic profiling. J Clin Oncol. 2011;29(22):3085-3096.

11. Poulikakos PI, et al. RAF inhibitor resistance is mediated by dimerization of aberrantly spliced BRAF(V600E). Nature. 2011;480(7377):387-390.

12. Flaherty KT, et al. Combined BRAF and MEK inhibition in melanoma with BRAF V600 mutations. N Engl J Med. 2012;367(18):1694-1703.

13. Kim KB, et al. Phase II study of the MEK1/MEK2 inhibitor Trametinib in patients with metastatic BRAF-mutant cutaneous melanoma previously treated with or without a BRAF inhibitor. J Clin Oncol. 2013;31(4):482-489.

14. Villanueva J, et al. Acquired resistance to BRAF inhibitors mediated by a RAF kinase switch in melanoma can be overcome by cotargeting MEK and IGF-1R/PI3K. Cancer Cell. 2010;18(6):683-695.

15. Yadav V, et al. Reactivation of mitogen-activated protein kinase (MAPK) pathway by FGF receptor 3 (FGFR3)/Ras mediates resistance to vemurafenib in human B-RAF V600E mutant melanoma. J Biol Chem. 2012;287(33):28087-28098.

16. Paraiso KHT, et al. PTEN loss confers BRAF inhibitor resistance to melanoma cells through the suppression of BIM expression. Cancer Res. 2011;71(7):2750-2760.

17. Shao Y, Aplin AE. BH3-only protein silencing contributes to acquired resistance to PLX4720 in human melanoma. Cell Death Differ. 2012; 19(12):2029-2039.

18. Gembarska A, et al. MDM4 is a key therapeutic target in cutaneous melanoma. Nat Med. 2012; 18(8):1239-1247.

19. Moon RT, et al. Xwnt-5A: a maternal Wnt that affects morphogenetic movements after overexpression in embryos of Xenopus laevis. Dev Camb Engl. 1993;119(1):97-111.

20. Weeraratna AT. A Wnt-er wonderland--the complexity of Wnt signaling in melanoma. Cancer

Address correspondence to: Randall T. Moon, HHMI and Department of Pharmacology, University of Washington School of Medicine, 850 Republican Street, Seattle, Washington 98109, USA. Phone: 206.543.1722; Fax: 206.616.4230; E-mail: rtmoon@uw.edu. Or to: Andy J. Chien, Division of Dermatology, University of Washington School of Medicine, Institute for Stem Cell and Regenerative Medicine, Campus Box 358056, 815 Mercer Street, Seattle, Washington 98109, USA. Phone: 206.616.4240; Fax: 206-543-1183; E-mail: andchien@uw.edu.

Metastasis Rev. 2005;24(2):237-250.

21. Bordonaro M, Tewari S, Cicco CE, Atamna W, Lazarova DL. A switch from canonical to noncanonical Wht signaling mediates drug resistance in colon cancer cells. PLoS One. 2011;6(11):e27308.

22. Kobune M, et al. Wnt3/RhoA/ROCK signaling pathway is involved in adhesion-mediated drug resistance of multiple myeloma in an autocrine mechanism. Mol Cancer Ther. 2007;6(6):1774-1784.

23. Zhang $\mathrm{H}$, et al. Interference of Frizzled 1 (FZD1) reverses multidrug resistance in breast cancer cells through the Wnt/ $\beta$-catenin pathway. Cancer Lett. 2012;323(1):106-113.

24 . Biechele TL, et al. Wnt $/ \beta$-catenin signaling and AXIN1 regulate apoptosis triggered by inhibition of the mutant kinase BRAFV600E in human melanoma. Sci Signal. 2012;5(206):ra3.

25. Flaherty KT, Hodi FS, Fisher DE. From genes to drugs: targeted strategies for melanoma. Nat Rev Cancer. 2012;12(5):349-361.

26. Davies MA. The role of the PI3K-AKT pathway in melanoma. Cancer J Sudbury Mass. 2012;18(2):142-147.

27. Tap WD, et al. Pharmacodynamic characterization of the efficacy signals due to selective BRAF inhibition with PLX4032 in malignant melanoma. Neoplasia N Y N. 2010;12(8):637-649.

28. O'Connell MP, et al. Hypoxia induces phenotypic plasticity and therapy resistance in melanoma via the tyrosine kinase receptors ROR1 and ROR2. Cancer Discov. 2013;3(12):1378-1393.

29. Smalley KSM, Lioni M, Herlyn M. Life isn't flat: taking cancer biology to the next dimension. In Vitro Cell Dev Biol Anim. 2006;42(8-9):242-247.

30. Chien AJ, et al. Activated Wnt/beta-catenin signaling in melanoma is associated with decreased proliferation in patient tumors and a murine melanoma model. Proc Natl Acad Sci U S A. 2009; 106(4):1193-1198.

31. Yang P-T, et al. WLS inhibits melanoma cell proliferation through the $\beta$-catenin signalling pathway and induces spontaneous metastasis. EMBO Mol Med. 2012;4(12):1294-1307.

32. Li J, et al. WNT5A antagonizes WNT/beta-catenin signaling and is frequently silenced by promoter CPG methylation in esophageal squamous cell carcinoma. Cancer Biol Ther. 2010;10(6):617-624.

33. Roarty K, Baxley SE, Crowley MR, Frost AR, Serra R. Loss of TGF- $\beta$ or Wnt5a results in an increase in Wnt/ $\beta$-catenin activity and redirects mammary tumour phenotype. Breast Cancer Res BCR. 2009;11(2):R19.

34. Biechele TL, Adams AM, Moon RT. Transcriptionbased reporters of Wnt $\beta$-catenin signaling. Cold Spring Harb Protoc. 2009;2009(6):pdb.prot5223.

35. Ying J, et al. WNT5A exhibits tumor-suppressive activity through antagonizing the Wnt/ $\beta$-catenin signaling, and is frequently methylated in colorectal cancer. Clin Cancer Res OffJ Am Assoc Cancer Res. 2008;14(1):55-61.

36. Huang S-MA, et al. Tankyrase inhibition stabilizes axin and antagonizes Wnt signalling. Nature. 2009;461(7264):614-620.

37. Dissanayake SK, et al. The Wnt5A/protein kinase $\mathrm{C}$ pathway mediates motility in melanoma cells via the inhibition of metastasis suppressors and initiation of an epithelial to mesenchymal transition. J Biol Chem. 2007;282(23):17259-17271.
38. Weeraratna AT, et al. Wnt5a signaling directly affects cell motility and invasion of metastatic melanoma. Cancer Cell. 2002;1(3):279-288.

39. O'Connell MP, et al. The orphan tyrosine kinase receptor, ROR2, mediates Wnt5A signaling in metastatic melanoma. Oncogene. 2010;29(1):34-44.

40. Vlahos CJ, Matter WF, Hui KY, Brown RF. A specific inhibitor of phosphatidylinositol 3-kinase, 2-(4-morpholinyl)-8-phenyl-4H-1-benzopyran-4-one (LY294002). J Biol Chem. 1994;269(7):5241-5248.

41. Gopal YNV, et al. Basal and treatment-induced activation of AKT mediates resistance to cell death by AZD6244 (ARRY-142886) in Braf-mutant human cutaneous melanoma cells. Cancer Res. 2010;70(21):8736-8747.

42. Su F, et al. Resistance to selective BRAF inhibition can be mediated by modest upstream pathway activation. Cancer Res. 2012;72(4):969-978.

43. Deng W, et al. Role and therapeutic potential of PI3K-mTOR signaling in de novo resistance to BRAF inhibition. Pigment Cell Melanoma Res. 2012;25(2):248-258.

44. Atefi M, et al. Reversing melanoma cross-resistance to BRAF and MEK inhibitors by co-targeting the AKT/mTOR pathway. PLoS One. 2011;6(12):e28973.

45. Baryawno N, et al. Small-molecule inhibitors of phosphatidylinositol 3-kinase/Akt signaling inhibit Wnt/ $\beta$-catenin pathway cross-talk and suppress medulloblastoma growth. Cancer Res. 2010;70(1):266-276.

46. Sánchez-Hernández I, Baquero P, Calleros L, Chiloeches A. Dual inhibition of (V600E)BRAF and the PI3K/AKT/mTOR pathway cooperates to induce apoptosis in melanoma cells through a MEK-independent mechanism. Cancer Lett. 2012;314(2):244-255.

47. Shao Y, Aplin AE. Akt3-mediated resistance to apoptosis in B-RAF-targeted melanoma cells. Cancer Res. 2010;70(16):6670-6681.

48. Angers S, Moon RT. Proximal events in Wnt signal transduction. Nat Rev Mol Cell Biol. 2009; 10(7):468-477.

49. Kikuchi A, Yamamoto H, Sato A, Matsumoto S. Wnt5a: its signalling, functions and implication in diseases. Acta Physiol Oxf Engl. 2012;204(1):17-33.

50. Bittner M, et al. Molecular classification of cutaneous malignant melanoma by gene expression profiling. Nature. 2000;406(6795):536-540.

51. Da Forno PD, et al. WNT5A expression increases during melanoma progression and correlates with outcome. Clin Cancer Res Off Am Assoc Cancer Res. 2008;14(18):5825-5832.

52. Tímár J, Gyorffy B, Rásó E. Gene signature of the metastatic potential of cutaneous melanoma: too much for too little? Clin Exp Metastasis. 2010;27(6):371-387.

53. Gentile A, Lazzari L, Benvenuti S, Trusolino L, Comoglio PM. Ror1 is a pseudokinase that is crucial for Met-driven tumorigenesis. Cancer Res. 2011;71(8):3132-3141.

54. Zhang $\mathrm{S}$, et al. ROR1 is expressed in human breast cancer and associated with enhanced tumor-cell growth. PLoS One. 2012;7(3):e31127.

55. Berndt JD, et al. Mindbomb 1, an E3 ubiquitin ligase, forms a complex with RYK to activate Wnt/ $\beta$-catenin signaling. J Cell Biol. 2011;194(5):737-750.

56. Hoek KS, et al. Metastatic potential of melanomas 
defined by specific gene expression profiles with no BRAF signature. Pigment Cell Res. 2006;19(4):290-302.

57. Lin WM, et al. Modeling genomic diversity and tumor dependency in malignant melanoma. Cancer Res. 2008;68(3):664-673.

58. Griesmann H. WNT5A-NFAT signaling mediates resistance to apoptosis in pancreatic cancer. $\mathrm{NeO}$ plasia. 2013;15(1):11-22.

59. Gowrishankar K, et al. Acquired resistance to BRAF inhibition can confer cross-resistance to combined BRAF/MEK inhibition.J Invest Dermatol. 2012;132(7):1850-1859.

60. Jiang CC, et al. MEK-independent survival of B-RAFV600E melanoma cells selected for resistance to apoptosis induced by the RAF inhibitor PLX4720. Clin Cancer Res. 2011;17(4):721-730.

61. Straussman R, et al. Tumour micro-environment elicits innate resistance to RAF inhibitors through HGF secretion. Nature. 2012;487(7408):500-504.

62 . Wilson TR, et al. Widespread potential for growthfactor-driven resistance to anticancer kinase inhibitors. Nature. 2012;487(7408):505-509.

63. Witze ES, Litman ES, Argast GM, Moon RT, Ahn NG. Wnt5a control of cell polarity and directional movement by polarized redistribution of adhesion receptors. Science. 2008;320(5874):365-369.

64. Von Maltzahn J, Bentzinger CF, Rudnicki MA Wnt7a-Fzd7 signalling directly activates the Akt/ mTOR anabolic growth pathway in skeletal muscle. Nat Cell Biol. 2011;14(2):186-191.

65. King TD, Zhang W, Suto MJ, Li Y. Frizzled7 as an emerging target for cancer therapy. Cell Signal. 2012;24(4):846-851.

66. Yang L, et al. FZD7 has a critical role in cell pro- liferation in triple negative breast cancer. Oncogene. 2011;30(43):4437-4446

67. Ueno K, et al. Down-regulation of frizzled-7 expression decreases survival, invasion and metastatic capabilities of colon cancer cells. BrJ Cancer. 2009;101(8):1374-1381.

68. Ueno K, et al. Frizzled-7 as a potential therapeutic target in colorectal cancer. Neoplasia. 2008;10(7):697-705.

69. Gurney A, et al. Wnt pathway inhibition via the targeting of Frizzled receptors results in decreased growth and tumorigenicity of human tumors. Proc Natl Acad Sci U S A. 2012;109(29):11717-11722.

70. Anastas JN, et al. A protein complex of SCRIB, NOS1AP and VANGL1 regulates cell polarity and migration, and is associated with breast cancer progression. Oncogene. 2012;31(32):3696-3708 